\title{
Experiência na definição e implantação do processo de análise e tomada de decisão alinhado ao CMMI-SW
}

\author{
Fca. Márcia G. S. Gonçalves, Solange Alcântara Araújo, Fabiana Marinho, Tatiana \\ Cavalcanti, Carlo Giovano Pires
}

\author{
Instituto Atlântico - SPD - Suporte ao Processo de Desenvolvimento \\ Rua Chico Lemos, 946 - Cidade dos Funcionários - CEP 60.822 - 780 - \\ Fone 55 (85) 3216- 7800 - Fortaleza - CE - Brasil \\ \{marcia, solange, fabiana, tatiana, cgiovano\} @atlantico.com.br
}

\begin{abstract}
One of the difficulties in the CMMI-SW level 3 implementation consists in defining and institutionalizing the Decision Analysis and Resolution process area because it is new and not commonly used in many organizations. The formal analysis reduces the subjectivity and move towards the selection of a solution that maximizes the attendance of stakeholder's necessities. In this context, this work shows the experience of an organization in Decision Analysis and Resolution process definition and implementation.
\end{abstract}

Resumo. Uma das dificuldades na implantação do CMMI-SW nível 3 consiste em definir e institucionalizar a área de processo de Análise e Tomada de Decisão por ser pouco conhecida e não utilizada em grande parte das instituições. A tomada de decisão formal reduz a subjetividade e é mais propensa a selecionar uma solução que maximize o atendimento das necessidades dos envolvidos. Diante deste contexto, este trabalho mostra a experiência de uma organização na definição e implantação do Processo de Tomada de Decisão Formal.

\section{Introdução}

Atualmente, muitas organizações ignoram a relevância de um processo formal para a tomada de decisão. No entanto, ela se faz importante para a melhoria de processo, apoio à visão da organização e objetivos de negócio, reduzindo a subjetividade da decisão e dando uma maior probabilidade de selecionar a solução que mais atende as múltiplas demandas dos envolvidos, podendo também ser aplicada para questões não técnicas [CMMI, 2002].

As tomadas de decisão formal podem ser feitas em várias situações, como por exemplo, seleção de fornecedores, priorização de orçamentos, avaliação de riscos de aquisições, soluções técnicas, abordagens de testes, design, entre outros. Vale ressaltar que as decisões formais devem ser feitas apenas para decisões significativas e com critérios claros para aplicação.

Os principais fatores que motivam as tomadas de decisão formal envolvem discussões sem convergência, falta de base para análise da situação, falta de objetividade, pontos de vista distintos, decisões e tomadas de decisão através de influências, análises parciais de problemas e impactos. 
Os pilares para as tomadas de decisão formal consistem na convergência da decisão com base na perspectiva e habilidades dos diversos envolvidos, avaliação das alternativas com base em critérios claros e objetivos, utilização de metodologias e técnicas como ferramentas de convergência para conduzir a discussão. O processo de convergência e análise da situação pode ser mais rico do que próprio resultado final, uma vez que as alternativas são analisadas pelos principais envolvidos, destacando pontos fortes e fracos de cada alternativa, chegando a um senso comum e por muitas vezes gerando novas alternativas [Kulpa, 2003].

Diante deste contexto, este trabalho visa compartilhar a experiência na definição e implantação do Processo de Análise e Tomada de Decisão (Decision Analysis and Resolution - DAR) que faz parte da Arquitetura de Processos do Instituto Atlântico, instituição avaliada no nível 3 de maturidade do modelo CMMI-SW em fevereiro de 2006.

Este artigo está estruturado em quatro seções. A seção 2 descreve o CMMI, destacando a área de processo de análise e tomada de decisão. O estudo de caso da definição e implantação do processo é apresentado na seção 3 e, a seção 4 , mostra as considerações finais deste trabalho.

\section{Análise e tomada de decisão no CMMI}

A área de processo (PA) de Análise e Tomada de Decisão compõe o nível 3 do CMMI-SW representação por estágios. Seu objetivo é analisar possíveis decisões usando um processo de avaliação formal que considere as alternativas identificadas em relação aos critérios definidos [CMMI, 2002]. Esta PA possui apenas um objetivo específico e seis práticas específicas, conforme vemos a seguir:

\section{- SG 1: Avaliar Alternativas}

○ SP 1.1 - Estabelecer Diretrizes para Análise da Decisão

- SP 1.2 - Estabelecer Critérios de Avaliação

○ SP 1.3 - Identificar Soluções Alternativas

○ SP 1.4 - Selecionar Métodos de Avaliação

○ SP 1.5 - Avaliar Alternativas

○ SP 1.6 - Selecionar Soluções

A seleção dos métodos de avaliação das soluções (SP 1.4) é crucial para o sucesso na institucionalização do processo de análise e tomada de decisão, merecendo uma atenção especial.

Métodos para avaliar soluções alternativas de acordo com critérios estabelecidos incluem simulações para o uso de modelos probabilísticos e decisões teóricas. Estes modelos precisam ser cuidadosamente selecionados. O nível de detalhes do método deve ser adequado para os custos, cronograma, desempenho e impacto dos riscos. Enquanto muitos problemas podem necessitar somente um método de avaliação, alguns problemas requerem múltiplos métodos. 
Observamos oportunidades do uso de tomadas de decisão formal em várias PA's do CMMI-SW e para cada situação faz-se necessária a análise do método de avaliação da solução mais adequado. Algumas destas oportunidades estão descritas a seguir.

- Project Monitoring and Control (PMC): ocorrência de problemas no projeto, seleção da ação a ser tomada;

- Supplier Agreement Management (SAM): decisão de "make-or-buy" e seleção de fornecedores;

- Measurement and Analysis (MA): seleção e priorização das métricas;

- Technical Solution (TS): seleção de alternativas de implementação dos requisitos e decisão de "make-buy-reuse";

- Product Integration (PI): identificação da sequiência ideal de integração;

- Organizational Process Focus (OPF): seleção de melhorias de processos;

- Organizational Training (OT): definição do tipo de treinamento e se será desenvolvido ou comprado.

- Risk Management (RSKM): seleção de ações para mitigar e contingenciar riscos;

\section{Estudo de caso}

O Processo de Análise e Tomada de Decisão do Instituto Atlântico tem como finalidade padronizar o processo de tomada de decisão formal, visando a sua transparência e objetividade, preservando o histórico e as bases sobre as quais as decisões são tomadas.

O fluxo do processo de Análise e Tomada de Decisão é considerado bastante simples, sendo composto por apenas duas macroatividades: Analisar Situação e Tomar Decisão, conforme podemos ver na Figura 1. O processo foi definido como uma disciplina do RUP [RUP, 2002] e descreve o fluxo das atividades que vão desde planejamento até tomada efetiva de decisão. 


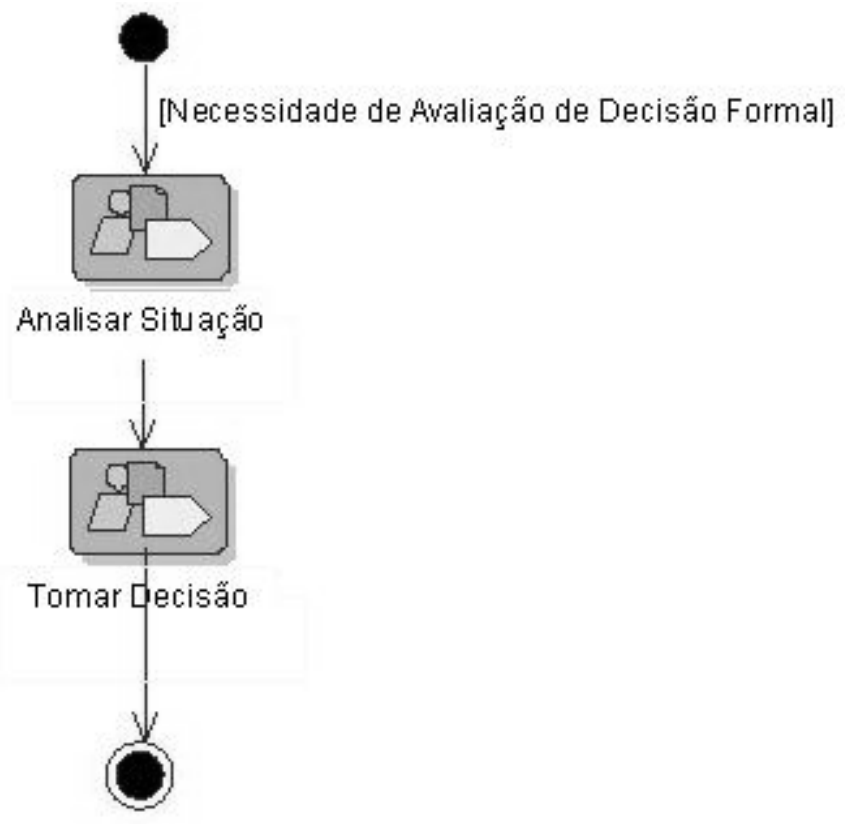

Figura 1 - Fluxo do Processo de Análise e Tomada de Decisão

Na macroatividade Analisar Situação, os principais pontos abordados são:

- Analisar necessidade de análise e tomada de decisão formal: consiste em avaliar se uma situação requer análise formal para tomada de decisão. Nem toda situação irá necessitar um processo formal, sua importância é variável de acordo com o contexto do projeto. Exemplos de situações que requerem decisão formal são: decisões tecnológicas e de arquitetura, COTS, seleção de fornecedores e tratamento de riscos.

- Estabelecer critérios: consiste na definição de critérios e orientações para a tomada de decisão. São exemplos de critérios pré-definidos: custo, prazo, escopo, qualidade e satisfação do cliente. Os critérios específicos dos projetos são aqueles relacionados a limitações tecnológicas, restrições geral do projeto e cliente, custo do ciclo de vida do produto e exposição a riscos. Cada critério deve ter um peso associado.

- Identificar e documentar as alternativas: consiste em identificar as alternativas para decisão, tendo por base as normas e práticas da organização, lições aprendidas de outros projetos, conhecimentos e habilidades dos envolvidos, situações semelhantes na organização, busca na literatura e mercado, critérios de avaliação e combinação de alternativas.

- Selecionar o método de avaliação: consiste em métodos utilizados para avaliação de alternativas envolvidas na tomada de decisão. $\mathrm{O}$ uso de um método tem como objetivo reduzir ou eliminar a subjetividade da tomada de decisão. A seção 3.1 descreve os métodos de avaliação adotados pela organização.

Na macroatividade Tomar Decisão são feitas as seguintes atividades: 
- Analisar as alternativas: consiste em avaliar alternativas com base no método selecionado.

- Selecionar a alternativa: consiste em, através do método de avaliação, tomar a decisão e selecionar a alternativa mais indicada. A seleção de uma alternativa pode servir de entrada para a análise de ações e atividades de planejamento ou ações corretivas para execução da solução.

$\mathrm{Na}$ tabela abaixo é apresentado um mapeamento entre as macroatividades do Processo de Análise e Tomada de Decisão e as práticas requeridas da PA de Decision Analysis and Resolution do CMMI-SW.

Tabela 1 - Mapeamento entre as macroatividades do processo e as práticas do CMMI-SW

\begin{tabular}{|l|l|l|}
\hline Macroatividade & \multicolumn{1}{|c|}{ Finalidade } & \multicolumn{1}{|c|}{ Prática CMMI-SW } \\
\hline Analisar Situação & $\begin{array}{l}\text { Analisar situações e planejar tomada de } \\
\text { decisão. }\end{array}$ & $\begin{array}{l}\text { SP 1.1 - Estabelecer diretrizes para } \\
\text { análise da decisão } \\
\text { SP 1.2 - Estabelecer critérios de } \\
\text { avaliação } \\
\text { SP 1.3 - Identificar soluções } \\
\text { alternativas } \\
\text { SP 1.4 - Selecionar métodos de } \\
\text { avaliação } \\
\text { GP 2.2 Planejar o processo }\end{array}$ \\
\hline Tomar Decisão & $\begin{array}{l}\text { Avaliar as alternativas identificadas e } \\
\text { selecionar a melhor opção com base nos } \\
\text { critérios definidos. }\end{array}$ & $\begin{array}{l}\text { SP 1.5 - Avaliar alternativas } \\
\text { SP 1.6 - Selecionar soluções }\end{array}$ \\
\hline
\end{tabular}

Conforme podemos verificar na tabela acima, todas as práticas específicas e uma prática genérica de DAR foram atendidas pelo Processo de Análise e Tomada de Decisão apresentado. As demais práticas genéricas são atendidas por outros processos e pela Política Institucional da Qualidade, de acordo com a Tabela 2.

Tabela 2. Mapeamento entre as práticas do CMMI-SW e os processos da organização

\begin{tabular}{|l|l|}
\hline \multicolumn{1}{|c|}{ Prática CMMI-SW } & \multicolumn{1}{c|}{ Política/ Processo } \\
\hline GP 2.1 Estabelecer uma política organizacional & Políticas Institucionais da Qualidade \\
\hline GP 2.3 Prover recursos & Processo de Gestão de Projetos \\
\hline GP 2.4 Atribuir responsabilidades & Processo de Gestão de Projetos \\
\hline GP 2.5 Treinar pessoas & Processo de Treinamento \\
\hline GP 2.6 Gerenciar as configurações & Processo de Gestão de Configuração e Mudança \\
\hline GP 2.8 Monitorar e controlar o processo & Processo de Gestão de Projetos \\
\hline GP 2.9 Avaliar a aderência de forma objetiva & Processo de Garantia da Qualidade \\
\hline GP 2.10 Revisar o status com a gerência de alto nível & Processo de Gestão de Projetos \\
\hline GP 3.1 Estabelecer um processo definido & $\begin{array}{l}\text { Processo de Gestão de Processos e Processo de } \\
\text { Gestão de Projetos }\end{array}$ \\
\hline GP 3.2 Coletar informações de melhoria & Processo de Gestão de Processos \\
\hline
\end{tabular}


O processo de Análise e Tomada de Decisão do Atlântico prevê algumas situações onde seu uso é obrigatório, como por exemplo, na definição da solução técnica da arquitetura, seleção de COTS e riscos de alta exposição podendo ser usado também em outras situações.

Caso seja identificada a necessidade de decisão formal, as atividades devem ser planejadas. O planejamento deve permitir:

- Estruturação e definição do método de avaliação;

- Definição dos envolvidos na tomada de decisão;

- Identificação de alternativas e critérios;

- Definição de ações, datas, recursos e eventos específicos;

- Seleção dos métodos de avaliação.

\subsection{Métodos de avaliação}

A organização possui atualmente três métodos de avaliação para realização das tomadas de decisão formal, são eles: método de critérios com valor ponderado, relatório técnico (survey) e prova de conceito. Outros métodos de avaliação podem ser utilizados pelos projetos, nesses casos é exigida a revisão e aprovação do Engineering Process Group (EPG).

\subsubsection{Método do valor ponderado}

O método de critérios com valor ponderado consiste no método padrão utilizado pela organização por sua adequação a várias situações, podendo ser utilizado na análise de riscos, análise de fornecedores e estratégias de integração. Esse método pode ser utilizado com uma variação utilizando notas negativas para pesar questões como pontos fortes e pontos fracos.

Inicialmente são selecionados os participantes cujo envolvimento é relevante para a decisão. Após o entendimento da situação que gerou a necessidade de tomada de decisão, os envolvidos definem um conjunto de critérios, justificando sua necessidade, e atribuem um peso para cada critério, variando numa escala de 0 a 5, conforme podemos ver no exemplo da Figura 2, que trata do uso do processo para uma situação de seleção de profissional para compor equipe de projeto.

\begin{tabular}{|l|c|c|}
\hline \multicolumn{1}{|c|}{ Tabela - Critérios } & Justificativa & Peso \\
\hline Formaçẫo & importante para o trabalho na instituiçẫo & 3 \\
\hline Disponiblidade de inicio & importante devido à urgência do projeto & 4 \\
\hline Conhecimento em Java & essencial para as atividades desempenhadas & 5 \\
\hline Certificaçấo & demonstra interesse em atualizaçẫo & 2 \\
\hline Conhecimento em RuP & importante para o trabalho na instituiçẫo & 2 \\
\hline Conhecimento em Casos de uso & necessário para especificar UC no projeto & 4 \\
\hline $\begin{array}{l}\text { Conhecimento em frameworks } \\
\text { Java }\end{array}$ & desejável para eficiência no projeto & 3 \\
\hline Personalidade & importante para a convivência & 3 \\
\hline Experiência profissional & importante para a empresa & 3 \\
\hline
\end{tabular}

Figura 2 - Exemplo de Critérios com Justificativas e Peso 
Em seguida, os envolvidos buscam alternativas para a decisão e atribuem as notas para cada alternativa proposta, conforme podemos ver no exemplo da Figura 3. O resultado é obtido a partir da média ponderada para cada alternativa.

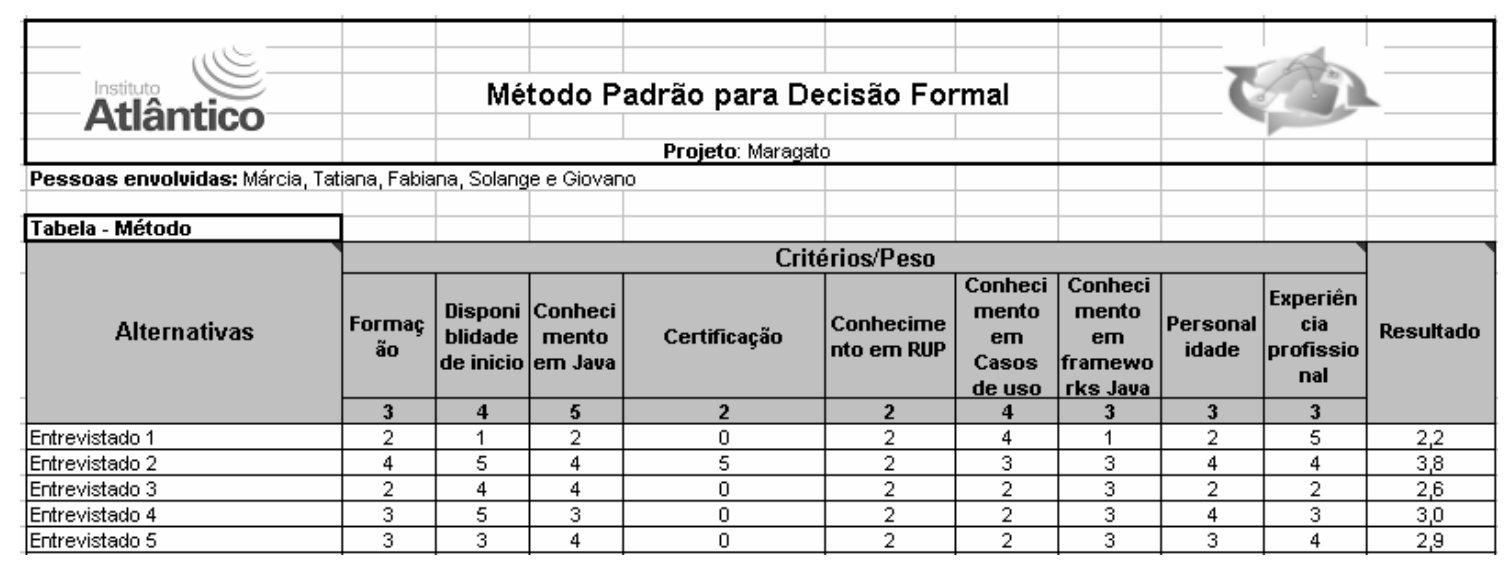

Figura 3 - Exemplo de Tabela de Alternativas, Critérios e Pesos para a Decisão Formal

As Figuras 2 e 3 nos mostram um exemplo de tomada de decisão para "Seleção de dois novos analistas de sistemas para a equipe do projeto" que resultou na contratação do "Entrevistado 2" e "Entrevistado 4" que obtiveram os melhores resultados dentre os candidatos.

\subsubsection{Relatório técnico (Survey)}

Método utilizado tipicamente para questões técnicas, nas quais alternativas são pesquisadas e avaliadas de forma qualitativa contra critérios com base em análise de especialistas e/ou avaliações de mercado e acadêmicas. Indicada para decisões tecnológicas e avaliação de componentes. O método é comumente utilizado em conjunto com outros para fornecer a devida estruturação e descrição dos critérios e alternativas adotados.

\subsubsection{Provas de conceito}

As provas de conceito (PoCs) consistem em um método baseado em simulação e prototipação e têm por objetivo testar uma determinada arquitetura de software, arquitetura de infra-estrutura de TI ou conceitos e funcionalidades de uma tecnologia específica. As PoCs também podem ser utilizadas para a automação de processos, isto é, definição de algoritmos que solucionam problemas de negócios de determinadas áreas da empresa.

De acordo com o MSF (Microsoft Solutions Framework) [MSF, 2002], cada PoC segue uma metodologia de projeto composta de cinco etapas: visão e escopo, planejamento, desenvolvimento, estabilização e implantação, podendo ser utilizadas em conjunto com o Método de Valor Ponderado ou Relatório Técnico. Como resultado final de uma PoC, os participantes recebem toda a documentação elaborada, abrangendo o planejamento da solução, plano de testes, especificação funcional, além de todo o código gerado. $\mathrm{O}$ conhecimento sobre as tecnologias aplicadas também é transferido para todos os participantes. 


\section{Conclusões}

A definição do Processo de Análise e Tomada de Decisão inicialmente pareceu muito difícil devido à falta de conhecimento, limitação de referencial teórico e exemplos de uso em outras organizações. Sendo assim, o apoio de uma consultoria especializada e treinamentos no modelo foram fundamentais para o entendimento desta PA.

A primeira versão do processo estava bastante complexa e as diretrizes definiam tomadas de decisão formal para inúmeras situações, a maioria delas inviáveis. Estas diretrizes foram revisadas durante as consultorias e foram sendo excluídas, resultando em um processo bastante simples.

Durante a institucionalização, observamos um certo receio das equipes dos projetos que achavam o processo burocrático para uma simples tomada de decisão. Neste momento, foi fundamental o apoio da alta gerência mostrando a importância e benefícios advindos de um processo formalizado, visto que alguns métodos de avaliação já eram bastante utilizados na organização.

Posteriormente, observamos que o processo já estava sendo usado até para situações que não haviam sido previamente definidas, o que comprovou que a real importância estava sendo dada à existência de um processo de análise e tomada de decisão formal.

O que inicialmente pareceu burocrático, veio a facilitar as tomadas de decisão, evitando discussões entre os responsáveis pelas decisões que defendiam seus pontos de vista, dificultando a convergência e aceitação da decisão. Além disso, tivemos um maior engajamento de outras pessoas na tomadas de decisão, facilitando a difusão do conhecimento e comprometimento, pois eles viam que suas idéias estavam sendo levadas em consideração, mesmo que não tenha sido a solução escolhida.

\section{Referências bibliográficas}

CMMI Product Team. (2002) "Capability Maturity Model Integration, version 1.1. CMMI for Software Engineering (CMMI-SW/IPPD, v1.1) Staged Representation”, Software Engineering Institute.

Kulpa, Margaret K. and Johnson, Kent A. (2003) "Interpreting the CMMI: a process improvent approach", Auerbach.

Process Group (2005) "Decision Analysis and Resolution Process" http://www.processgroup.com/pgpostoct05.pdf. Acessado em 05/03/2006.

MSF Process Model v. 3.1 (2002) http://www.microsoft.com/msf. Acessado em 08/03/2006.

RUP “Rational Unified Process Tutorial”. Versão 20020500. 